It is a document of great importance to the future of the Survey and of India. Sewell makes it clear that an enlarged Survey is essential to the scientific and economic advancement of the country. There should be a very considerable expansion of the staff. Originally there were four officers, then seven, now only five. There should be at least two dozen in the near future, so that each branch of the animal kingdom shall have its expert. One of the new officers would be naturalist to the Marine Survey. This would be a revival of the surgeon-naturalist's post, so fruitful in the past in the deep-sea work of R.I.M.S. Investigator.

The immediate difficulty will be to fill these zoological posts. Early this year I was in India as a delegate to the Indian Science Congress and then visited a number of the universities. Some of the zoology departments were excellent, others less good; but in most of them the standard could well be raised. In Indian universities zoology has not been so much developed as some of the other sciences. This is, no doubt, a matter of tradition, hard to change. In addition there are in India very few field naturalists. This means that first-class personnel for expanding the Survey will not easily be found. Sewell has put forward a scheme for the training of young Indian zoological graduates of the universities for two years in the Survey and then for a year abroad.

The Survey, with all its valuable collections and library, was moved for safety during the War from Calcutta to temporary quarters at Benares. There it is to-day, quite inadequately housed. There is insufficient room either to tend or to consult the collections, and the building is liable to floods which have already damaged valuable books. The Survey has the best zoological library in India, probably in Asia. Sewell recommends the erection at New Delhi of a new home for the Survey in a fire-proof, air-conditioned building. Here it would be placed centrally for Government consultation, situated near other scientific institutes.

When India assumes independence she cannot afford to neglect the expansion of her Zoological Survey.

\section{NEW PHYSICO-CHEMICAL PHENO- MENA IN THE DEFORMATION AND MECHANICAL TREATMENT OF SOLIDS*}

\section{By P. REHBINDER}

Member of the Academy of Sciences of the U.S.S.R.

$\mathrm{T}$ HE mechanical structural properties of solidselasticity, plasticity and strength-are of paramount importance in most branches of modern engineering. These properties, which are determined by molecular interaction, are usually investigated without resorting to advanced physical chemistry. However, the methods of physico-chemical investigation of the deformation of solids, with due recourse to the factor of the chemical composition of the surrounding medium, lead to new methods of controlling the mechanical properties of solids and to novel methods of their mechanical treatment.

* Summary of a report at a general meeting of the Academy of Sciences of the U.S.S.R. in July 1946.
The earlier investigations by the author and his associates have established the fact that the addition to the surrounding medium of small amounts of the so-called surface-active substances, the particles of which are adsorbed on solid surfaces, is capable of considerably lowering the resistance of a solid to stress.

These very general phenomena of facilitating strain and disintegration of solids by adsorption have been extensively utilized by the author and his co-workers, and afterwards by many other investigators, both in the Soviet Union and abroad in various practical fields. For example, they have been used to facilitate the boring of stable (hard) rocks, in various processes of disintegration of solids by milling, in cutting and pressure working of metals, etc. It is this phenomenon of adsorptional promotion of strain that largely accounts for the effect of cooling lubricants, in the absence of which some of the above processes become altogether impracticable. The theory of this phen. omenon must underlie any scientifically based selection of these lubricants.

During the Second World War, mainly in connexion with the application of these investigations to the machining of metals, we succeeded in elucidating to some extent the mechanism of the adsorptional promotion of strain and, at the same time, in discovering a number of new phenomena in this domain.

It was ascertained that these phenomena are associated with the penetration of surface-active substances into the depth of the strained solid along the surface of the wedge-shaped ultra-microscopic cracks which are developed in course of deformation. After unloading, that is, removal of the stress, the micro-cracks become closed again, the surface-active substances adsorbed on their inner surface are expelled by molecular cohesion and the original mechanical properties of the solid are restored.

The closing of the micro-cracks after unloading is a process involved not only in elastic but also in plastic deformations, with the sole difference that, after shear involving slippage, the closing of the cracks takes place at new points of their surfaces.

These investigations have led to the discovery of some new phenomena which have been described in the report referred to above, and partly demonstrated in a micro-cinematographic film. Special interest is presented by two effects resulting from the penetration of surface-active substances into the microcracks: the retarded elastic deformation in mica crystals and the structural changes on plastic deformation of metals.

When a sheet of mica is bent or stretched in absolutely dry air, the deformation occurs practically instantaneously as soon as the external force is applied, and disappears also instantaneously after removal of the stress when the latter is below the elastic limit. In the presence of moisture, however -in water vapour or when the erystal is wetted with water-a gradual increase of deformation on application of stress is observed and a gradual decrease on removal of the stress. On addition to the water of organic substances readily adsorbed on mica, for example, alcohols, this retardation is still further enhanced, and the time required for the deformation to attain its maximum or to disappear after unloading is a matter of some three days. Complete recovery is observed in the case of these phenomena.

So long ago as in the experiments of I. Obreimov on the cleaving of mica along the cleavage planes in 
vacuo, it was demonstrated that the cleft formed by introduction of a glass wedge is closed again on removal of the latter, so that subsequent eleavage requires almost the same amount of mechanical work. This phenomenon underlies the ingenious method suggested by I. Obreimov for measuring the surface tension at the cleavage faces of mica. The cleavage of mica in such experiments is quite similar to the formation of wedge-shaped micro-cracks, which, according to our concepts, are developed in the region of elastic deformations without any rupture of the solid. As has been kindly pointed out to us by Prof. I. Frenkel, such a concept of reversibly closing micro-cracks leads to the elimination of some grave flaws in Griffith's well-known theory and opens new ways towards its substantial improvement.

In studying the plastic flow in single crystals of the purest tin, it was established that under the action of the surface-active substances (for example, oleic acid or cetyl alcohol) added to the surrounding inert medium (purified paraffin oil), the number of the layers formed which slip over each other on extension of the single crystal increases fifteen-fold or more, whereas their thickness decreases in proportion. These phenomena were observed by means of accelerated micro-cinemaphotography of the stretched single crystals. The sorption of the organic surfaceactive substances by the strained metal lowers the yield value of tin to half the original value, facilitating the flow by a kind of 'internal' lubrication of the slip planes. Such sorption manifests itself in the abrupt fall of the electrical conductivity of tin, which on removal of the external stress is gradually restored as the original mechanical properties of the metal are restored.

In the case of ordinary, small-grained zinc and copper, the strain-hardening of the outer layers was found to increase sharply after periodic deformation in the presence of surface-active substances. The adsorptional lowering of the hardness of the metal originally observed on deformation is followed by its sharp increase due to a secondary phenomenon : strong disintegration of the crystal grains or their orientation (formation of texture), which always leads to hardening. Some new electrocapillary phenomena were also observed and investigated, namely, the lowering of the hardness or strength of metals in solutions of electrolytes when the surfaces of the micro-cracks formed are charged electrically, irrespective of the sign of charge.

The seat of the above peculiar surface phenomena is thus the inner surface of the micro-cracks which are developed in a strained solid. The latter is, as it were, a kind of labile adsorbent, the newly formed surface of which gradually disappears again after unloading. The whole set of phenomena of adsorptional promotion of deformation in a solid can be regarded as evidence in favour of our concepts regarding the continuous development of microcracks in the process of deformation, beginning from the region of elastic deformations.

The elucidation of these phenomena is evidence of the fruitful result of resorting to physical chemistry in the problems of physics and mechanics of solids. This boundary domain of science can in general be said to be of great practical importance in the solution of the problems involved in the working and machining of metals and other solids, as well as in the theory of wear, creep, corrosion, fatigue and a number of other problems in the applied physics of metals and building materials.

\section{THE FIRST CHEMICAL JOURNAL}

\author{
By Prof. JAMES KENDALL, F.R.S.
}

TN connexion with the forthcoming centenary celebrations (postponed from 1941) of the Chemical Society, the discovery of the first volume of the proceedings of an older organisation in the same field is of timely interest.

This volume a well-bound folio of 452 pages in copper-plate manuscript, inscribed simply "Chemical Society instituted in the beginning of the year 1785" -was presented to the Royal Irish Academy by Sir William Betham on January 26, 1846, and lay unnoticed in its library for more than a century. Recently, however, it attracted the attention of the Rev. P. J. McLaughlin, of St. Patrick's College, Maynooth, and through an article written by me in the July 1942 number of Endeavour, Dr. Farrington, librarian of the Academy, and Dr. McLaughlin were able to identify it as Volume 1 of the Proceedings of the Chemical Society of the University of Edinburgh. Practically all the thirty-two communications which it records in extenso represent, in fact, papers read at meetings of this Society by members whose names are on Joseph Black's "List of the Members of the Chemical Society, 1785".

That it should have found a resting-place in Dublin is not really surprising. The nationality of nineteen of the fifty-nine original members of the Edinburgh society has been definitely established; of these only three were native Scots, three English, and the residual thirteen were Irish ! Presumably the unknown secretary, to whose admirable diligence the volume owes its existence, was one of this majority and retained possession of his handiwork on his return to Ireland.

At my request, the Council of the Royal Irish Academy agreed to send the volume on loan to the Royal Society of Edinburgh for my inspection. Unfortunately, the communications do not include anything of major scientific importance, and my expectation that the volume might afford a unique record of contemporary chemical thought has not been realized. I had hoped to find some lively discussions (with Joseph Black himself participating) on the revolution that Lavoisier was then leading against the phlogiston theory, but this is rarely even mentioned. Most of the papers are routine descriptive chemistry of the classical period, and the most promising of the few exceptions, "an attempt [by Mr. Thomas Beddoes, later director of the Pneumatic Institution and supervisor of Humphry Davy] to point out some of the consequences which flow from $\mathrm{Mr}$. Cavendishes discovery of the component parts of water" peters out, after an attractive start, into an addendum entitled "a conjecture concerning the use of Manure" !

This disappointment, however, emboldened me to miake a second request to the Council of the Royal Irish Academy, that it should present the volume to its original owners, and to that request it has now graciously consented. The oldest chemical society in the world will certainly treasure the first volume of its proceedings as a priceless possession. Even if it has no great intrinsic value, it does after all constitute the first journal extant of a purely chemical character, the Annales de Chimie being initiated just five years later; and extracts from it may well prove worthy of wider publication. 